\title{
Right Bochdalek Hernia Associated with Kartagener Syndrome: Developmental and Clinical Observations
}

\author{
Carmelo Romeo $^{1}$ Nunzio Turiaco ${ }^{1}$ Eloisa Gitto ${ }^{1}$ Francesca Astra Borruto $^{1}$ Giuseppe Santoro ${ }^{2}$ \\ ${ }^{1}$ Department of Pediatrics, Gynecology, Microbiology, and Biomedical \\ Sciences, Policlinico G. Martino, University of Messina, Messina, Italy \\ 2 Department of Biomorphology and Biotechnologies, Policlinico G. \\ Martino, University of Messina, Messina, Italy \\ Pediatrics, Gynecology, Microbiology, and Biomedical Sciences, \\ University of Messina, Via Consolare Valeria, 1, Messina 98125, Italy \\ (e-mail: romeoc@unime.it).
}

Eur J Pediatr Surg Rep 2013;1:15-17.

\begin{abstract}
Keywords

- Bochdalek hernia

- Kartagener syndrome

- diaphragm development

We present a novel case of the association of right-sided Bochdalek hernia, a diaphragmatic life-threatening malformation, and Kartagener syndrome, which is characterized by congenital bronchiectasis, chronic sinusitis, and situs inversus. The developmental and clinical findings are discussed. When an association of diaphragmatic hernia with situs viscerum inversus is encountered, physicians should be mindful of the possibility of Kartagener syndrome because this condition could significantly affect the morbidity of the patient.
\end{abstract}

\section{Introduction}

Congenital posterolateral diaphragmatic hernia $(\mathrm{CDH})$ or Bochdalek hernia $(\mathrm{BH})$ is a life-threatening malformation characterized by an anomaly of the posterolateral foramina of Bochdalek, through which the abdominal viscera migrate into the chest during fetal development. ${ }^{1}$ The incidence of $\mathrm{CDH}$ is 1 in 2,500 to 3,000 newborns. ${ }^{2,3}$ Vertebrates exhibit evolutionarily conserved asymmetries in the pattern of internal organ placement, which are essential for normal physiological function. Left-right asymmetries in organ situs are dependent on the formation of an intact left-right axis during embryogenesis. Alterations in the classic asymmetries (situs solitus) may occur as a complete mirror image reversal of all of the organs (situs inversus totalis), or an incomplete reversal resulting in a wide spectrum of intermediate defects (situs ambiguus), including heterotaxy (reversal of individual organs along the left-right axis) and isomerism (duplication or changes in normal symmetry). In situs inversus totalis, the heart is located on the right side of the chest, the left lung is trilobed and the right lung is bilobed, and the stomach and spleen occupy the right side, while the liver and gallbladder are present on the left side of the abdomen and the intestine is transposed. ${ }^{3}$ The situs inversus (SI) condition has an estimat- ed occurrence of 1 in 5,000 to 20,000 births and is either undiagnosed or is diagnosed incidentally during the investigation of another associated aliment. ${ }^{4,5}$ One-fourth to onefifth of patients with SI have a genetically heterogeneous, autosomal-recessive disorder known as primary ciliary dyskinesia (PCD), a dysfunction of the cilia that manifests during embryologic development. ${ }^{3,6}$ Normal functioning cilia are critical to the embryological development of proper organ laterality, and approximately 50\% of PCD patients present with abnormalities of thoracoabdominal symmetry. ${ }^{6}$ These patients have Kartagener syndrome (KS), initially reported in the early 1900s, which is characterized by the triad of SI, chronic sinusitis, and bronchiectasis. ${ }^{7}$ To the best of our knowledge, this is the first report documenting a case of a right-sided $\mathrm{BH}$ in a patient with $\mathrm{KS}$.

\section{Case Report}

The report focuses on a $4.180-\mathrm{kg}$ baby boy born by cesarian section from an epileptic mother, with the end of a gestation complicated by threatened abortion and polyhydramnios. Soon after birth, the boy developed acute respiratory distress and cyanosis. Physical examination revealed a distended thorax, intercostal retractions with decreased breathing received

November 20, 2012

accepted after revision

January 30, 2013

published online

April 15, 2013 (c) 2013 Georg Thieme Verlag KG

Stuttgart · New York
DOI http://dx.doi.org/ 10.1055/s-0033-1343077. ISSN 2194-7619. 


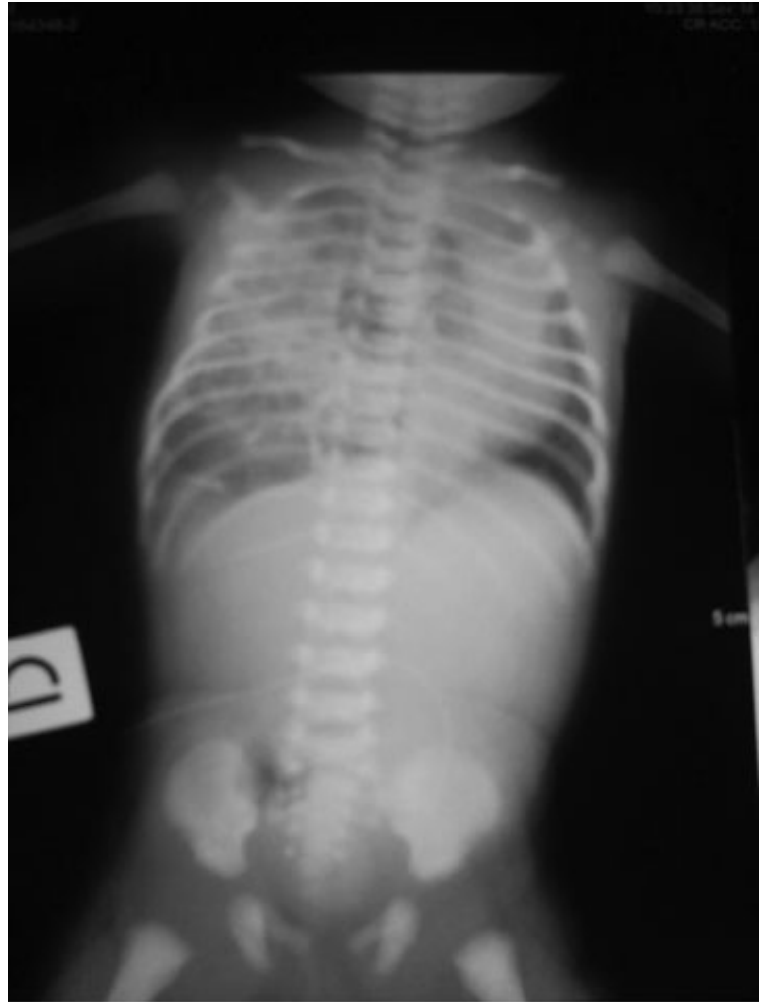

Fig. 1 Chest radiograph documenting right diaphragmatic hernia.

sounds on the right side of the thorax, and a scaphoid abdomen. A chest radiograph revealed the presence of intestinal loops in the right pleural cavity with left displacement of the mediastinum ( - Fig. 1). The baby was initially stabilized by emergency endotracheal intubation and ventilated with synchronized intermittent positive pressure ventilation with the following parameters: Ti 0.3, Te 1, PEEP 4, and $\mathrm{FIO}_{2} 35 \%$. After 48 hours of stabilization, the baby underwent a laparotomy through the right abdomen. The right diaphragmatic defect was exposed and was sutured with interrupted nonabsorbable sutures. During laparotomy, the liver and the gallbladder appeared in the left and the spleen in the right hypochondrium. Therefore, SI was suspected. The postoperative course was complicated by drainage of the right pneumothorax. The baby was weaned from ventilation on the 7th postoperative day. A chest X-ray ( - Fig. 2), echocardiography, and electrocardiograms revealed a right-sided heart; the barium meal documented greater curvature of the rightoriented stomach and the barium enema showed a mirror image of intestinal tube, thereby confirming SI. The baby was discharged on the 15th postoperative day with medical therapy for gastroesophageal reflux. During follow-up, the baby had several episodes of bronchitis and otitis. He underwent examinations to exclude cystic fibrosis, which included a sweat test and a genetic test that revealed the lack of relevant mutations. In vitro and electron microscopic ultrastructural analyses of nasal scraping respiratory cilia confirmed KS. A tympanogram revealed a flat curve, and parental history of SI was negative.

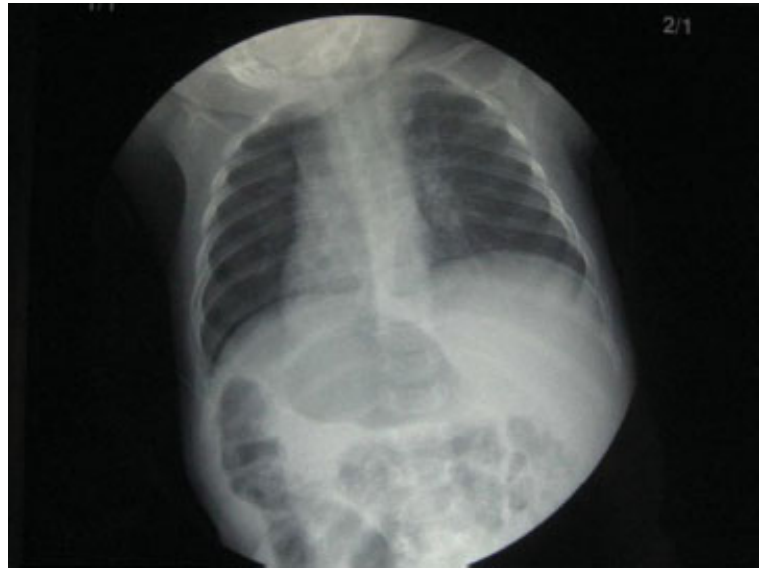

Fig. 2 Postoperative chest radiograph documenting dextrocardia.

\section{Discussion}

Right-sided $\mathrm{BH}$ and KS are two rare disorders, with no reported association. Embryogenesis of $\mathrm{BH}$ has been described as a failure in the closure of the pericardioperitoneal canal by the pleuroperitoneal membranes, which occurs during gestational week 8 th. $^{8}$ For this reason, it is hypothesized that an early return in the coelomic cavity of the foregut results in wide posterolateral spaces and dysfunctional pleuroperitoneal membranes. Consequently, the abdominal viscera herniate into the thorax, which is thought to cause pulmonary hypoplasia by compression of the growing lung. ${ }^{8}$ The left $\mathrm{BH}$ is the most frequent $\mathrm{CDH}$, and its preponderance with respect to the right side may be related to the early closure of the right pleuroperitoneal opening, although no theories have been postulated to explain this. ${ }^{9} \mathrm{CDH}$ can be associated with different syndromes, such as Fryns, DonnaiBarrow, Beckwith-Wiedemann, Sympson-Golabi-Behmel, Coffin-Siris, and Denys-Drash syndromes. However, the association with KS has never been described. ${ }^{10} \mathrm{KS}$ is characterized by the triad of SI, chronic sinusitis, and bronchiectasis and is a subset of one of the most widely recognized ciliopathies, PCD, in which ciliary motility defects occur. Particularly during embryogenesis, KS could alter normal left-right axis formation, which determines the SI. Sutherland and Ware have recently offered a detailed view of the five steps necessary to establish asymmetry in the vertebrate. SI, caused by PCD, usually results from defects in signaling at the node involving cilia structure and function. ${ }^{10}$ Three previous reports have described single laterality abnormalities in combination with a right-sided diaphragmatic defect; only one has shown an abdominal SI associated with a right-sided $\mathrm{BH}$ in which the karyotype was normal and the family history mentioned no other cases of laterality defects. ${ }^{3}$ Retinoic acid has been implicated in both diaphragmatic development and situs anomalies. However, no evidence exists in the literature regarding the role of retinoic acid in KS. Thus, the two malformations in the present case are considered an occasional occurrence. 


\section{Conclusions}

This is the first case of the association of right-sided BH and KS in a live patient. When dealing with an association of diaphragmatic hernia with situs inversus totalis, physicians should be mindful of the potential contribution of ciliary dyskinesia to chronic respiratory disease (particularly bronchiectasis), which might otherwise be attributed to gastroesophageal reflux disease. Multidisciplinary clinical management of both malformations with early identification and treatment is warranted.

\section{Conflict of Interest}

None

\section{References}

1 Chiu P, Hedrick HL. Postnatal management and long-term outcome for survivors with congenital diaphragmatic hernia. Prenat Diagn 2008;28(7):592-603

2 Peetsold MG, Heij HA, Kneepkens CMF, Nagelkerke AF, Huisman J, Gemke RJ. The long-term follow-up of patients with a congenital diaphragmatic hernia: a broad spectrum of morbidity. Pediatr Surg Int 2009;25(1):1-17

3 Tesselaar CD, Postema RR, van Dooren MF, Allegaert K, Tibboel D. Congenital diaphragmatic hernia and situs inversus totalis. Pediatrics 2004;113(3, Pt 1):e256-e258

4 Borgaonkar VD, Deshpande SS, Kulkarni VV. Laparoscopic cholecystectomy and appendicectomy in situs inversus totalis: a case report and review of literature. J Minim Access Surg 2011;7 (4):242-245

5 Tayeb M, Khan FM, Rauf F. Situs inversus totalis with perforated duodenal ulcer: a case report. J Med Case Reports 2011; $5: 279$

6 Kennedy MP, Omran H, Leigh MW, et al. Congenital heart disease and other heterotaxic defects in a large cohort of patients with primary ciliary dyskinesia. Circulation 2007;115(22):2814-2821

7 Kartagener M. Zur pathogenese der bronkiectasien bei situs viscerum inversus. Beitr Klin Tuberk 1933;82:489-501

8 Puri P, Nakazawa N. Congenital diaphragmatic hernia. In: Puri P, Hollwarth M, eds. Pediatric Surgery: Diagnosis and Management. Berlin, Heidelberg: Springer Verlag; 2009:307-314

9 Moore KL, Persaud TVN. The Developing Human. Clinically Oriented Embryology. 8th ed. Philadelphia, PA: WB Saunders; 2008:150-158

10 Sutherland MJ, Ware SM. Disorders of left-right asymmetry: heterotaxy and situs inversus. Am J Med Genet C Semin Med Genet 2009;151C(4):307-317 